\title{
Translational tilings of the integers with long periods
}

\author{
Mihail N. Kolountzakis* \\ Department of Mathematics, \\ University of Crete, \\ Knossos Ave., 71409 Iraklio, \\ Greece.
}

\section{E-mail: kolount@member.ams.org}

Submitted: Oct 31, 2002; Accepted: May 8, 2003; Published: May 12, 2003 MR Subject Classifications: Primary 11B75, Secondary 10A25

\begin{abstract}
Suppose that $A \subseteq \mathbb{Z}$ is a finite set of integers of diameter $D=\max A-\min A$. Suppose also that $B \subseteq \mathbb{Z}$ is such that $A \oplus B=\mathbb{Z}$, that is each $n \in \mathbb{Z}$ is uniquely expressible as $a+b, a \in A, b \in B$. We say then that $A$ tiles the integers if translated at the locations $B$ and it is well known that $B$ must be a periodic set in this case and that the smallest period of $B$ is at most $2^{D}$. Here we study the relationship between the diameter of $A$ and the least period $\mathcal{P}(B)$ of $B$. We show that $\mathcal{P}(B) \leq c_{2} \exp \left(c_{3} \sqrt{D} \log D \sqrt{\log \log D}\right)$ and that we can have $\mathcal{P}(B) \geq c_{1} D^{2}$, where $c_{1}, c_{2}, c_{3}>0$ are constants.
\end{abstract}

\section{Notation.}

Let $G$ be an abelian group denoted additively.

If $A, B \subseteq G$ we write

$$
A+B=\{a+b: \quad a \in A, b \in B\}
$$

We also write $A+b$ in place of $A+\{b\}$ to denote a translate of set $A$.

If in the set $A+B$ every element is written uniquely as $a+b$, with $a \in A$ and $b \in B$ we may write $A \oplus B$ in place of $A+B$.

If $A, B, E \subseteq G$ and $E=A \oplus B$ we say that $A$ tiles $E$ when translated by $B$ (and similarly that $B$ tiles $E$ when translated by $A$ ). We also say that $A \oplus B$ is a tiling of $E$ by $A$ (or $B$ ). Sets $A$ for which there is $B \subseteq G$ such that $G=A \oplus B$ are called tiles.

*Supported in part by European Commission IHP Network HARP (Harmonic Analysis and Related Problems), Contract Number: HPRN-CT-2001-00273 - HARP. 
For every positive integer $n$ we write $[n]=\{0,1, \ldots, n-1\}$. Subsets of $[n]$ will also be viewed as subsets of $\mathbb{Z}_{n}=\mathbb{Z} /(n \mathbb{Z})$, the additive group of residues $\bmod n$.

For any integer $n$ and a set $X \subseteq G$ we write

$$
n X=\{n x: \quad x \in X\} .
$$

A set $B \subseteq G$ is called periodic if there exists a nonzero $g \in G$ such that $B+g=B$. Such a $g$ is then called a period of $B$ and clearly the set of all periods plus 0 forms a, possibly trivial, subgroup of $G$. In case $B \subseteq \mathbb{Z}$, is a periodic set of integers, its least positive period is denoted by $\mathcal{P}(B)$. The set $B$ is then clearly a union of congruence classes $\bmod \mathcal{P}(B)$ and we can write

$$
B=\widetilde{B} \oplus \mathcal{P}(B) \mathbb{Z},
$$

where $\widetilde{B} \subseteq[\mathcal{P}(B) \mathbb{Z}]$.

\section{Introduction and results}

It has long been known (see e.g. [7]) that, when $G=\mathbb{Z}$ is the integer group, $A \oplus B=\mathbb{Z}$ and $A \subseteq \mathbb{Z}$ is a finite set, then $B$ is necessarily periodic. We are interested in the case when we know the diameter $D$ of the tile $A$, and ask how large can the period of a tiling by $A$ be. (The period of a tiling by a finite set $A$ when translated by $B$ is the quantity $\mathcal{P}(B)$.) In $[7]$ it is proved that every such tiling has period at most $2^{D}$.

The main quantity of interest is defined below.

Definition 1 If $D$ is a positive integer we write $\mathcal{T}(D)$ for the largest integer $k$ such that there exists $A \subseteq\{0, \ldots, D\}$ and $B \subseteq \mathbb{Z}$ with $\mathcal{P}(B)=k$ and $A \oplus B=\mathbb{Z}$.

It has been noted by some authors (e.g. [2]) that no tilings $A \oplus B=\mathbb{Z}$ are known with $\mathcal{P}(B)>2 D$ and $A \subseteq\{0, \ldots, D\}$. So apparently the state of knowledge regarding the function $\mathcal{T}(D)$ is

$$
2 D \leq \mathcal{T}(D) \leq 2^{D},
$$

as it is easy to see that $2 D$ can be achieved for the tile $A=\{0, D\}$ which tiles with the set of translates $B=\{0, \ldots, D-1\}+(2 D) \mathbb{Z}$.

In this paper we improve these bounds somewhat, at least in the asymptotic sense.

Theorem 1 There are absolute constants $c_{1}, c_{2}, c_{3}>0$ such that, for $D>1$,

$$
c_{1} D^{2} \leq \mathcal{T}(D) \leq c_{2} \exp \left(c_{3} \sqrt{D} \log D \sqrt{\log \log D}\right) .
$$

Theorem 1 follows directly from Theorems 2 and 3 below.

Theorem 2 There is a constant $c_{1}>0$ such that for all $D>1$ there are tilings $X \oplus Y=\mathbb{Z}$ with $X \subseteq\{0, \ldots, D\}$ and

$$
\mathcal{P}(Y) \geq c_{1} D^{2} .
$$


Theorem 3 There are constants $c_{2}, c_{3}>0$ such that, for all $D>1$, if $A \oplus B=\mathbb{Z}$ is a tiling and $A \subseteq\{0, \ldots, D\}$ then

$$
\mathcal{P}(B) \leq c_{2} \exp \left(c_{3} \sqrt{D} \log D \sqrt{\log \log D}\right) .
$$

Remarks.

1. In Theorem 2 we have not been able to construct a set $X$ which tiles only with long periods. This would be very interesting as this could be a finitary and translational analog of aperiodic tiling, the ability that is of a set of tiles to tile space but only aperiodically [3].

2. There is a theorem of de Bruijn [1, Theorem 1] whose proof can be used to prove Theorem 2. De Bruijn constructs a non-periodic tiling in certain abelian groups. With proper choices of the parameters of his proof one can get, for arbitrarily large $N$, a cyclic group $\mathbb{Z}_{N}$ to be non-periodically tiled by a subset of it of diameter at most $\alpha N^{1 / 2}$, where $\alpha>0$ is a constant. This is the essential ingredient for the proof of Theorem 2, as is shown by Lemma 1 below. We think our proof is more intuitive (but a more general problem is studied in [1]).

3. The largest part of the proof of Theorem 3 appears also in an argument by Granville in $[5]$.

4. As pointed out to the author by S. Konyagin and an anonymous referee, and as proved independently by I. Ruzsa in [8], Theorem 3 (and, consequently, Theorem 1 ) is not sharp.Working a more carefully on the least common multiple instead of the product (see the proof of Theorem 3 below) one gets the upper estimate $c_{4} \exp \left(c_{5} \sqrt{D \log D}\right)$, for some positive constants $c_{4}$ and $c_{5}$.

5. I expect the lower bound in Theorem 1 to be closer to the true order of magnitude of $\mathcal{T}(D)$ than the upper bound.

Tiling the integers with a long period is equivalent to tiling a long finite cycle in a non-periodic way, as the following easy lemma claims.

Lemma 1 Suppose $A \subseteq[M], M$ a positive integer, and that $B \subseteq \mathbb{Z}$ has period $M$ : $B=\tilde{B} \oplus M \mathbb{Z}$, where $\tilde{B} \subseteq[M]$ and $\tilde{B}$ is viewed also a set in the cyclic group $\mathbb{Z}_{M}$. Then $A \oplus B=\mathbb{Z}$ and $M=\mathcal{P}(B)$ if and only if $A \oplus \tilde{B}=\mathbb{Z}_{M}$ and $\tilde{B}$ is not a periodic set in $\mathbb{Z}_{M}$.

Proof. Suppose that $A \oplus B=\mathbb{Z}$. It follows that $A \oplus(\tilde{B} \oplus M \mathbb{Z})=\mathbb{Z}$ hence $A \oplus \tilde{B}=$ $\mathbb{Z} / M \mathbb{Z}=\mathbb{Z}_{M}$. If $t \in\{1, \ldots, M-1\}$ and $\tilde{B}=\tilde{B}+t$ in $\mathbb{Z}_{M}$ then $B=(\tilde{B}+t)+M \mathbb{Z}=B+t$ which contradicts the fact that $M$ is a minimal period for $B$. Conversely, suppose that $A \oplus \tilde{B}=\mathbb{Z}_{M}$ and that $\tilde{B}$ is not a periodic set in $\mathbb{Z}_{M}$. Then $A \oplus B=A \oplus \tilde{B} \oplus M \mathbb{Z}=$ $[M] \oplus M \mathbb{Z}=\mathbb{Z}$ is a tiling of period $M$. Furthermore, by the previous argument if $B$ has a smaller period $t \in\{1, \ldots, M-1\}$ it follows that $\tilde{B}$ has $t$ as a period in $\mathbb{Z}_{M}$, a contradiction. 
However, it is not possible to have non-repetitive tilings of intervals with tiles which are very short compared to the interval. Essentially, in any tiling of an interval by a tile of diameter $D$ the length of the interval is at most $2 D$, otherwise there is a smaller sub-tiling. This fact is probably responsible for some researchers being unaware of "complicated" tilings (such as those claimed by Theorem 2), as one tends to think of tilings of the integers which are created by first tiling an interval and then repeating the tiling of that interval in order to tile the integer line.

Theorem 4 If $0 \in A \cap B, n>1, A \oplus B=[n]$ as subsets of $\mathbb{Z}$, and $\max A>\max B$ then $A$ is periodic as a subset of $\mathbb{Z}_{n}$.

Remark.

Under the assumptions of Theorem $4 \max A \neq \max B$, as the two sets cannot have two elements in common (0 and their common maximum) without violating unique representation in $A \oplus B$. So one of $\max A, \max B$ is larger.

Corollary 1 If $A \subseteq\{0, \ldots, D\}, 0 \in A \cap B, A \oplus B=[n]$ is a tiling in $\mathbb{Z}$ and $n>2 D$ there is $t<n$ such that $t \mid n$ and $A \oplus(B \cap[t])=[t]$ is already a tiling.

Proof of Corollary 1. Since $n-1=\max A+\max B$ it follows that $\max B>\max A$ and, by Theorem 4 with the roles of $A$ and $B$ reversed, $B=B+t$ in $\mathbb{Z}_{n}$, for some $t \in\{1, \ldots, n-1\}, t \mid n$. From this it follows that $A \oplus(B \cap[t])=[t]$ is also a tiling in $\mathbb{Z}$.

\section{Proofs}

Proof of Theorem 2. By Lemma 1 it suffices to construct, for all $D$ larger than a certain number $D_{1}$, a set $A \subseteq\{0, \ldots, D\}$ and a non-periodic set $B \subseteq \mathbb{Z}_{M}$, such that $A \oplus B=\mathbb{Z}_{M}$ is a tiling and $M \geq c_{6} D^{2}$, where $c_{6}>0$ is a constant that will be specified later in the proof. The constant $c_{1}$ then is taken small enough so as to have $\mathcal{T}(D) \geq c_{1} D^{2}$ even for $D \leq D_{1}$.

Assume that $D>D_{1}=10^{5}$ and pick $p$ and $q$ two distinct primes in the range $D / 200 \leq p, q \leq D / 50$, for example. Two such primes always exist. Let $M=2 \cdot 3 \cdot 5 \cdot p \cdot q$, so that $M \geq c_{6} D^{2}=\frac{30}{200^{2}} D^{2}$. The group $\mathbb{Z}_{M}$ is isomorphic to $\mathbb{Z}_{3 p} \times \mathbb{Z}_{5 q} \times \mathbb{Z}_{2}$, and we visualize it as a parallelogram $Q$ in three dimensions with $\mathbb{Z}_{3 p}$ along the $x$-axis, $\mathbb{Z}_{5 q}$ along the $y$-axis and $\mathbb{Z}_{2}$ along the $z$-axis, and keep in mind that opposite $x$ and $y$ edges are identified and that "moving" distance 1 upwards from the upper level brings one back to the lower level.

We define $A$ as being the $3 \times 5$ rectangle on the $x y$-plane with corner at 0 ,

$$
A=\{(i, j, 0): 0 \leq i<3,0 \leq j<5\},
$$

as is shown in Figure 1. Now we observe that $A$ can tile the group $Q$ non-periodically, as shown in Figure 2. To obtain the tiling shown in Figure 2, first tile $Q$ by $A$ in the usual way, i.e. with $B$ equal to the subgroup generated by the elements of $Q$ : $(3,0,0),(0,5,0)$ 


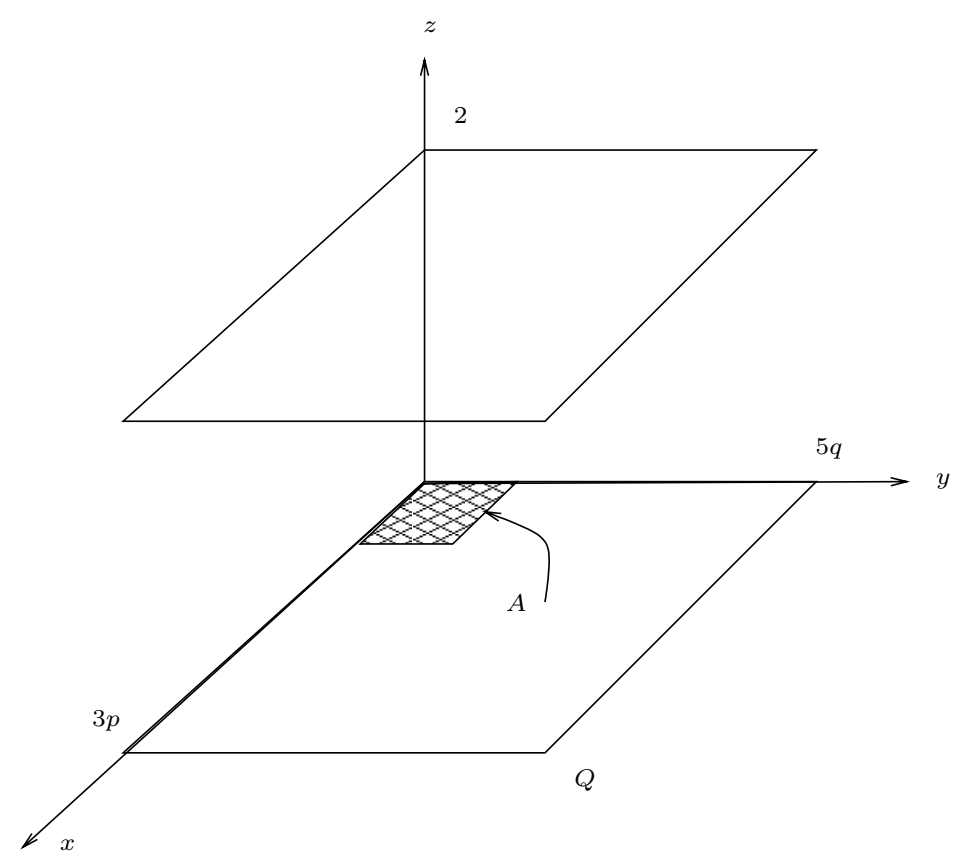

Figure 1: The group $\mathbb{Z}_{3 p} \times \mathbb{Z}_{5 q} \times \mathbb{Z}_{2}$

and $(0,0,1)$. Then, in the bottom level of the tiling shift one of the columns along the $x$ direction, say the second one as shown, by the vector $(1,0,0)$, and shift the second row in the upper level (one which is parallel to the $y$ direction) by the vector $(0,1,0)$.

The set $B$ is therefore given by $B=L \cup U$ (lower and upper part) and $L=\{(i, j, 0)\}$, where $j$ takes all values in $\langle 5\rangle \subseteq \mathbb{Z}_{5 q}$ and $i$ takes all values in $\left\langle 3>\subseteq \mathbb{Z}_{3 p}\right.$, except when $j=5$ in which case $i$ takes all values in $<3>+1 \subseteq \mathbb{Z}_{3 p}$. Similarly $U=\{(i, j, 1)\}$, where $i$ takes all values in $\left\langle 3>\subseteq \mathbb{Z}_{3 p}\right.$ and $j$ takes all values in $<5>\subseteq \mathbb{Z}_{5 q}$, except when $i=3$, in which case $j$ takes all values in $<5\rangle+1 \subseteq \mathbb{Z}_{5 q}$.

It is clear that $B$ is not periodic. Indeed, if $t=(i, j, k) \in Q \backslash\{0\}$ is a period then it must belong to $B$ as $0 \in B$. If $t \in L$ then it must belong to the group $<(3,0,0)\rangle$ but $U$ shifted by any of these non-zero elements does not go into $U$, because the second row shifted by such an element does not go into another row. If $t \in U$ then $U+t$ ought to be equal to $L$ but it is clearly not.

Fix now a group isomorphism $\psi: Q \rightarrow \mathbb{Z}_{M}$ and define

$$
X=\psi(A), \quad Y=\psi(B)
$$

Clearly $X \oplus Y=\mathbb{Z}_{M}$ is a tiling and $Y$ is not a periodic set in $\mathbb{Z}_{M}$, as both these properties are invariant under group isomorphisms. It remains to find the diameter of $X$, which of course depends on the choice of the isomoprhism $\psi$. Choose then the isomorphism

$\psi(i, j, k)=i(2 \cdot 5 q)+j(2 \cdot 3 p)+k(3 p 5 q) \bmod M, \quad(i=0, \ldots, 3 p-1, j=0, \ldots, 5 q-1, k=0,1)$, which maps $A$ to the set $X \subseteq\{0, \ldots, 20 q+24 p\}$. Hence the set $X$ has diameter at most 


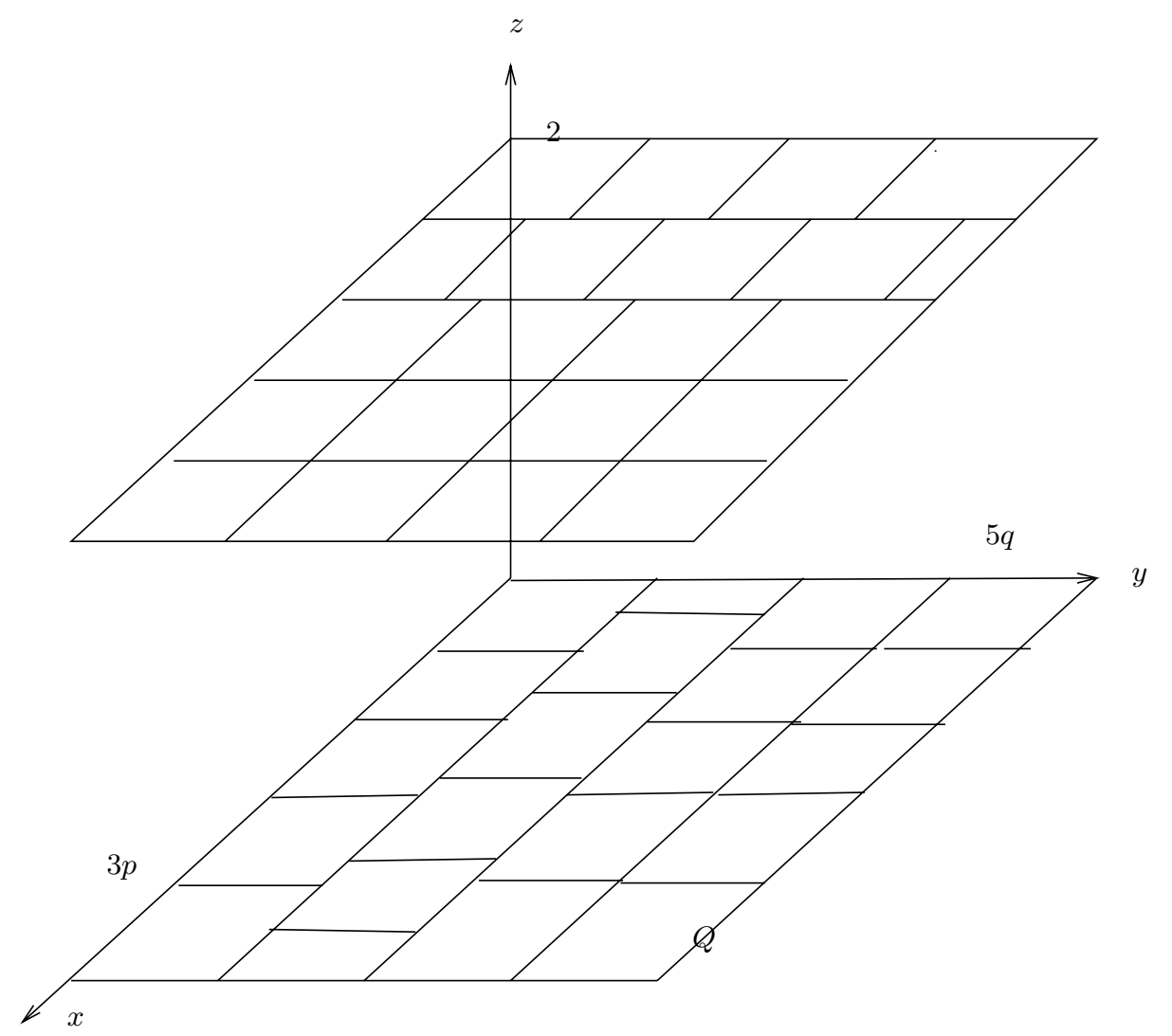

Figure 2: A non-periodic tiling by $A$

$\frac{44}{50} D \leq D$ whereas $M \geq c_{6} D^{2}$.

Remarks.

1. In our construction for the proof of Theorem 2 the tile $X$ has constant cardinality 15.

2. There is nothing magical about the choice of the primes 2,3 and 5 in the proof of Theorem 2. Any three different primes distinct from $p$ and $q$ could be used, but this does not allow one to get a larger order of magnitude for the lower bound.

Proof of Theorem 3. Suppose that $A \subseteq\{0, \ldots, D\}, D<M$, and that $A \oplus \tilde{B}=\mathbb{Z}_{M}$, for some $\tilde{B} \subseteq \mathbb{Z}_{M}$. We will show that if $M$ is large then $\tilde{B}$ is periodic. The Theorem follows using Lemma 1.

We use the cyclotomic polynomials which are the irreducible polynomials $\Phi_{s}(x), s=$ $1,2, \ldots$, defined by the equation

$$
x^{n}-1=\prod_{s \mid n} \Phi_{s}(x)
$$


valid for all $n=1,2, \ldots$ Equivalently $\Phi_{s}(x)$ is the minimal polynomial of any primitive $s$-th root of unity. The degree of $\Phi_{s}(x)$ is $\phi(s)$, the Euler function, i.e. the number of $t \in\{1, s\}$ which are coprime to $s$.

For $\widetilde{B}$ to be periodic we must show the existence of $t \in \mathbb{Z}_{M} \backslash\{0\}$ such that $\tilde{B}=\tilde{B}+t$. Equivalently, writing for any finite set of integers $E$

$$
E(x)=\sum_{n \in E} x^{n}
$$

we must ensure that

$$
\tilde{B}(x)=x^{t} \tilde{B}(x) \bmod x^{M}-1
$$

This is equivalent to

$$
x^{M}-1 \mid\left(x^{t}-1\right) \tilde{B}(x) .
$$

On the other hand, the fact that $A \oplus \tilde{B}$ is a tiling of $\mathbb{Z}_{M}$ is equivalent to

$$
A(x) \tilde{B}(x)=1+x+x^{2}+\cdots+x^{M-1} \bmod x^{M}-1,
$$

which is equivalent to

$$
x^{M}-1 \mid A(x) \tilde{B}(x)-\frac{x^{M}-1}{x-1},
$$

which implies that all $M$-th roots of unity except 1 are roots of $A(x)$ or $\tilde{B}(x)$. Equivalently, we must have that for each $d \mid M, d>1$, the cyclotomic polynomial $\Phi_{d}(x)$ divides $A(x)$ or $\tilde{B}(x)$. And, similarly, (2) is equivalent to having all cyclotomic polynomials $\Phi_{d}(x)$, for $d \mid M, d>1$, divide $x^{t}-1$ or $\tilde{B}(x)$.

Hence, for $t$ to be a period of $\tilde{B}$, it suffices that all cyclotomic $\Phi_{d}(x)$, for $d \mid M$, $d>1$, that divide $A(x)$ also divide $x^{t}-1$. Let now $\Phi_{s_{1}}(x), \ldots, \Phi_{s_{k}}(x)$ be all cyclotomic polynomials $\Phi_{s}(x)$ with $s>1$ that divide $A(x)$, written once each and numbered so that $1<s_{1}<s_{2}<\cdots<s_{k}$. Since $\operatorname{deg} \Phi_{s}=\phi(s)$ it follows that

$$
\phi\left(s_{1}\right)+\cdots+\phi\left(s_{k}\right) \leq \operatorname{deg} A(x) \leq D .
$$

But [4, p. 267]

$$
\phi(n) \geq c_{4} \frac{n}{\log \log n}, \quad\left(n \geq n_{0}\right),
$$

where $c_{4}$ can be taken equal to $e^{-\gamma}-\epsilon$ and $n_{0}$ is a constant $(\epsilon>0$ can be as small as we please if we enlarge $\left.n_{0}\right)$. Multiplying the above inequality by $\log \log n$ and taking logarithms we get

$$
\log \phi(n)+\log \log \log n \geq \log c_{4}+\log n
$$

and hence

$$
\log \phi(n) \leq \log n \leq 2 \log \phi(n), \quad\left(n>n_{1}>n_{0}\right),
$$

where $n_{1}$ is a constant. 
We have

$$
\begin{aligned}
& \sum_{i=1}^{k} s_{i}=\sum_{s_{i} \leq n_{1}} s_{i}+\sum_{s_{i}>n_{1}} s_{i} \\
& \leq n_{1}^{2}+\sum_{s_{i}>n_{1}}\left(e^{-\gamma}+\epsilon\right) \phi\left(s_{i}\right) \log \log s_{i} \\
& \leq n_{1}^{2}+\left(e^{-\gamma}+\epsilon\right) D \log \log s_{k} \\
& \leq n_{1}^{2}+\left(e^{-\gamma}+\epsilon\right) D(\log 2+\log \log D) \\
& \leq\left(e^{-\gamma}+2 \epsilon\right) D \log \log D \text {, } \\
& \text { (for } D>D_{2} \text { ) }
\end{aligned}
$$

where $D_{2}$ is a positive constant.

From the fact that the $s_{i}$ are different integers and the inequality

$$
s_{1}+\cdots+s_{k} \leq\left(e^{-\gamma}+2 \epsilon\right) D \log \log D, \quad\left(D>D_{2}\right)
$$

proved above it follows that

$$
k \leq c_{5} \sqrt{D \log \log D}, \quad\left(D>D_{2}\right)
$$

(where $c_{5}$ is a positive constant) hence

$$
\begin{aligned}
\prod_{i=1}^{k} s_{i} & \leq\left(\left(e^{-\gamma}+2 \epsilon\right) D \log \log D\right)^{k} \\
& \leq \exp \left(2 c_{5} \sqrt{D} \log D \sqrt{\log \log D}\right), \quad\left(D>D_{3}\right)
\end{aligned}
$$

where we used: $\log \left(\left(e^{-\gamma}+2 \epsilon\right) D \log \log D\right) \leq 2 \log D$ for $D>D_{3}$, a constant.

Define now $t=\prod_{i=1}^{k} s_{i}$, so that all cyclotomic polynomials that divide $A(x)$ are also divisors of $x^{t}-1$. From (7) it follows that (2) holds and, therefore, $t$ is a period of $\tilde{B}$, if only $t<M$. But $\tilde{B}$ is assumed to be non-periodic so

$$
M \leq c_{2} \exp \left(c_{3} \sqrt{D} \log D \sqrt{\log \log D}\right)
$$

as we had to prove, where $c_{3}=2 c_{5}$ and $c_{2}>1$ is taken large enough to make sure that (1) holds for all $D \leq D_{3}$ as well.

Proof of Theorem 4. We use the following result by Long [6, Lemma 1].

Theorem A Suppose $n>1,0 \in A \cap B$ and $A \oplus B=[n]$. Then there is an integer $m \geq 2$, $m \mid n$, and sets $E, D \subseteq[n / m]$, such that $E \oplus D=[n / m], 0 \in E \cap D$, and

$$
A=m E \oplus[m], B=m D
$$

or

$$
A=m D, B=m E \oplus[m] .
$$


Suppose now that $n>1$ is minimal such that Theorem 4 fails for $n$ and let $A, B \subseteq[n]$, $0 \in A \cap B$, be such that $\max A>\max B, A \oplus B=[n]$ and $A$ is not periodic as a subset of $\mathbb{Z}_{n}$. Now Theorem A applies and let $m, E, D$ be as in the conclusion of it. If $m=n$ it follows that $A=[n], B=\{0\}$, so that $A$ is periodic as a subset of $\mathbb{Z}_{n}$. If $m<n$ then $n>n / m>1$ hence Theorem 4 holds for the integer $n / m$.

Since $E \oplus D=[n / m]$ is a tiling it follows (see remark after Theorem 4) that $\max E \neq$ $\max D$ and there are now two cases: (i) $\max E>\max D$, and (ii) $\max D>\max E$.

In case (i) $\max (m E+[m])>\max (m D)$ hence $A=m E+[m]$. Since Theorem 4 holds for the integer $n / m$ it follows that $E$ is periodic as a subset of $\mathbb{Z}_{n / m}$ with some period $t \in\{1, \ldots, n / m-1\}$, which implies that $A$ is periodic as a subset of $\mathbb{Z}_{n}$ with period $m t \in\{1, \ldots, n-1\}$.

In case (ii) $\max (m D)>\max (m E+[m])$ hence $A=m D$. By Theorem 4 again we get that $D$ is periodic as a subset of $\mathbb{Z}_{n / m}$ with some period $t \in\{1, \ldots, n / m-1\}$, and $A$ is therefore periodic as a subset of $\mathbb{Z}_{n}$ with period $m t \in\{1, \ldots, n-1\}$.

In both cases we conclude that our theorem applies for $n$, hence it is true for all $n$.

Acknowledgment: I would like to thank the anonymous referees for their thorough work.

\section{Bibliography}

[1] N.G. de Bruijn, One the factorization of finite abelian groups, Nederl. Akad. Wetensch. Proc. Ser. A, 56 (Indagationes Math. 15) (1953), pp. 258-264.

[2] E.M. Coven and A. Meyerowitz, Tiling the integers with translates of one finite set, J. Algebra 212 (1999), no. 1, pp. 161-174

[3] B. Grünbaum and G.C. Shephard, Tilings and patterns. An introduction. A Series of Books in the Mathematical Sciences. W.H. Freeman and Company, New York, 1989.

[4] G.H. Hardy and E.M. Wright, An introduction to the theory of numbers, Fifth Edition, Oxford Univ. Press, 1978.

[5] S. Konyagin and I. Łaba, Spectra of certain types of polynomials and tiling of integers with translates of finite sets, preprint at arXiv math.NT/0209204 (2002).

[6] C.T. Long, Addition theorems for sets of integers, Pacific J. Math., 23 (1967), 1, pp. 107-112.

[7] D.J. Newman, Tesselations of integers, J. Number Th. 9 (1977), pp. 107-111.

[8] I. Ruzsa, Appendix in "R. Tijdeman, Periodicity and almost-periodicity, preprint, August 2002". 